\title{
Fibrina rica em plaquetas e leucócitos (L-PRF) em procedimentos de regeneração óssea guiada
}

\author{
Platelet- and leukocyte-rich fibrin (L-PRF) in guided bone regeneration procedures \\ Fibrina rica en plaquetas y leucocitos (L-PRF) en procedimientos de regeneración ósea guiada
}

Recebido: 05/08/2021 | Revisado: 10/08/2021 | Aceito: 30/08/2021 | Publicado: 22/09/2021

Caroline Liberato Marchiolli

ORCID: https://orcid.org/0000-0001-8881-4882 Universidade Brasil, Brasil

E-mail: caaliberato@gmail.com

Jéssica Monique Lopes Moreno

ORCID: https://orcid.org/0000-0002-8808-4872

Universidade Estadual Paulista "Júlio de Mesquita Filho", Brasil

E-mail: jessica_morenoo@hotmail.com

Ricardo Junior Denardi

ORCID: https://orcid.org/0000-0001-8312-7493

Universidade de São Paulo, Brasil

E-mail:ricardojdenardi@gmail.com

Caroline Martin Denardi

ORCID: https://orcid.org/0000-0003-2003-1712

Centro Universitário Avantis, Brasil

E-mail: cmartindenardi@gmail.com

Jennifer Lorrayne Bezerra Araujo Recalde

ORCID: https://orcid.org/0000-0002-3066-5703

Centro Universitário Avantis, Brasil

E-mail: jennifer.akrieger@yahoo.com

Wirley Gonçalves Assunção

ORCID: https://orcid.org/0000-0002-8903-0737

Universidade Estadual Paulista “Júlio de Mesquita Filho", Brasil

E-mail: wirley.assuncao@unesp.br

Ingrid Kely dos Reis Santos

ORCID: https://orcid.org/0000-0001-7726-1792

Centro Universitário Avantis, Brasil

E-mail:ingrrid_21@hotmail.com

Ricardo Alves Toscano

ORCID: https://orcid.org/0000-0002-6381-463X

Universidade Estadual Paulista "Júlio de Mesquita Filho", Brasil

E-mail: odontosc.joi@terra.com.br

\begin{abstract}
Resumo
O objetivo deste presente artigo é apresentar um caso clínico de regeneração óssea guiada com uso de fibrina rica em plaquetas e leucócitos L-PRF. Paciente do gênero feminino, ASA II, 72 anos, compareceu a clínica odontológica com queixa principal de acentuada mobilidade no elemento dental 24 (FDI). Após anamnese, exame clínico e exame tomográfico observou-se acentuada perda da tábua óssea vestibular e apical ao referido elemento dental. O plano de tratamento escolhido foi a exodontia, instalação imediata do implante e regeneração óssea guiada. Observou-se, no referido caso clínico, a integridade do substituto ósseo na região enxertada, assim como, a saúde e a integridade periimplantar.
\end{abstract}

Palavras-chave: Regeneração óssea guiada; Plaquetas; Implante dentário.

\begin{abstract}
The aim of this article is to present a clinical case of guided bone regeneration using platelet-rich fibrin and L-PRF leukocytes. A 72-year-old female patient, ASA II, came to the dental clinic with a chief complaint of severe mobility of tooth 24 (FDI). After anamnesis, clinical and tomographic examination we observed a marked loss of buccal and apical bone plate of the tooth. The treatment plan chosen was exodonty, immediate implant installation and guided bone regeneration. The integrity of the bone substitute in the grafted region, as well as the peri-implant health and integrity, were observed in this clinical case.
\end{abstract}

Keywords: Guided bone regeneration; Platelets; Dental implant. 


\section{Resumen}

El objetivo de este artículo es presentar un caso clínico de regeneración ósea guiada utilizando fibrina rica en plaquetas y leucocitos L-PRF. Paciente femenina, ASA II, 72 años, acudió a la clínica dental con la queja principal de una marcada movilidad en el elemento dental 24 (FDI). Tras la anamnesis, el examen clínico y tomográfico se observó una marcada pérdida de la placa ósea bucal y apical del diente. El plan de tratamiento elegido fue la exodoncia, la colocación inmediata del implante y la regeneración ósea guiada. En este caso clínico, se observó la integridad del sustituto óseo en la región injertada, así como la salud e integridad periimplantaria.

Palabras clave: Regeneración ósea guiada; Plaquetas; Implante dental.

\section{Introdução}

Nos estágios iniciais da cicatrização após intervenções cirúrgicas ou fraturas ósseas a cascata de coagulação ativa as plaquetas. O conteúdo dos grânulos das plaquetas é liberado no local da ferida, fornecendo simultaneamente uma infinidade de fatores de crescimento autólogos em proporções fisiológicas para o local afetado e contribuindo para o processo de cicatrização (Bosweel Et al.,2012).

Os agregados plaquetários facilitam o recrutamento, a proliferação e a maturação das células que participam da regeneração de tendões, ligamentos, ossos e cartilagens, podendo ser benéficos para tecidos com restrito suprimento sanguíneo em regiões de proliferação celular lenta. A fibrina rica em plaquetas (PRF - platelet-rich fibrin) desempenha um importante papel nos procedimentos cirúrgicos orais. Desde a sua descrição inicial por Choukroun em 2000, há muitos artigos científicos publicados até o momento sobre o assunto (Canellas Et al.,2018). Entre as indicações de utilização desse biomaterial destacamse: cirurgias de terceiro molar, preservação do rebordo após extrações dentárias, procedimentos de levantamento de seio maxilar, regeneração óssea guiada, reparo de fissuras alveolar, utilização concomitante com implantes dentários, tratamento cirúrgico para osteonecrose e tratamentos de comunicações oroantrais (Dohan Et 1.,2010). Evidência científicas mostram diminuição da prevalência de osteíte alveolar na utilização de fibrina rica em plaquetas (PRF) em cavidades de extração de terceiro molar. Em estudo comparativo de regeneração óssea avaliando a associação entre o PRF+biomaterial e utilização exclusiva de biomaterial mostrou, através da análise quantitativa, um aumento significativo da regeneração ósseo na associação de biomaterial ao PRF. Evidências científicas demonstram que a liberação de fatores de crescimento derivados do sangue facilita a angiogênese e aumenta o fluxo sanguíneo na cavidade sinusal favorecendo e acelerando a consolidação óssea em procedimentos de levantamento de membrana de seio maxilar (Zang Et al.,2012).

PRF é uma plaqueta pertencente a segunda geração de concentrados produzida sem a manipulação bioquímica do sangue. Em sua composição observa-se a presença de plaquetas e seus fatores de crescimento ativados que são substancialmente embutidos na matriz de fibrina durante o processo de polimerização natural. A presença de leucócitos e suas citocinas na PRF (L-PRF) contribuem para ação anti-infecciosa e a regulação da resposta imune no processo de cicatrização. Essa composição é beneficiada pela densidade e organização complexa da arquitetura matricial de fibrina que é produzida por uma polimerização natural sem a adição de qualquer agente anticoagulante ou gelificante (Dohan Et al.,2009). Essa forte arquitetura da fibrina distingue-se de outros tipos de concentrados como o plasma rico em plaquetas (PRP). Através da matriz de fibrina é possível a liberação lenta de fatores de crescimento durante o estágio de proliferação da cicatrização das feridas por um período de 7 a 14 dias. A presença de fibras finas com microporosidades podem servir como suporte para a migração celular. Esses concentrados contêm uma mistura de fatores anabólicos (derivados de plaquetas) e catabólicos (produzidos por leucócitos), embora suas concentrações variem em muitos sistemas de preparação diferentes (Kobayashi Et al.,2017). Estudo comparativo entre o L-PRP e o L-PRF mostrou nesse último maiores quantidades de liberação do fator de transformação de crescimento beta (TGF- $\boldsymbol{\beta})$. Sabe-se que a estrutura da rede de fibrina e a concentração de leucócitos influenciam a liberação de fator de crescimento nos concentrados de plaquetas (Pirraco et al.,2013). A coagulação rápida do L-PRP resultante da adição 
de trombina está em forte contraste com a coagulação lenta que ocorre no L-PRF e no coágulo sanguíneo nos quais permitem a liberação de fatores de crescimento e uma maior indução da migração celular. O PRF é um importante reservatório de fatores de crescimento endotelial vascular (VEGF). Além disso, existem grandes quantidades de fatores de crescimento derivados de plaquetas (PDGFs) nos grânulos das plaquetas que atuam como reguladores essenciais para a produção de colágeno além de migração e proliferação de células mesenquimais (Soldatos,E tal., 2017).

Evidências científicas mostram as membranas de PRF (L-PRF) podem ser cortadas em pequenos pedaços e quando adicionadas ao material de enxerto funcionam como uma matriz biológica promovendo a atração de células osteoprogenitoras para o centro do enxerto induzindo nova angiogênese (Simonpieri,Et al.,2009).

O objetivo deste presente artigo é apresentar um caso clínico de regeneração óssea guiada com uso de fibrina rica em plaquetas e leucócitos L-PRF.

\section{Metodologia e Relato de Caso}

Este artigo refere-se a um estudo com ênfase à aplicação de fibrina rica em plaquetas e leucócitos (L-PRF) em procedimentos de regeneração óssea guiada na implantodontia. Os procedimentos empregados neste trabalho seguiram os padrões éticos propostos pela Declaração de Helsinque de 1975, revisada em 2000. A paciente foi tratada após emissão do parecer de aprovado do comitê de Ética em Pesquisa do Centro Universitário Avantis -UNIAVAN-CEP/UNIAVAN e assinatura do termo de consentimento livre e esclarecido-TCLE.

Paciente do gênero feminino, ASA II, 72 anos, compareceu a clínica odontológica com queixa principal de acentuada mobilidade no elemento dental 24 (FDI). Após anamnese, exame clínico e exame tomográfico (Figura 1) observou-se acentuada perda da tábua óssea vestibular e apical ao referido elemento dental. O plano de tratamento escolhido foi a exodontia, instalação imediata do implante e regeneração óssea guiada.

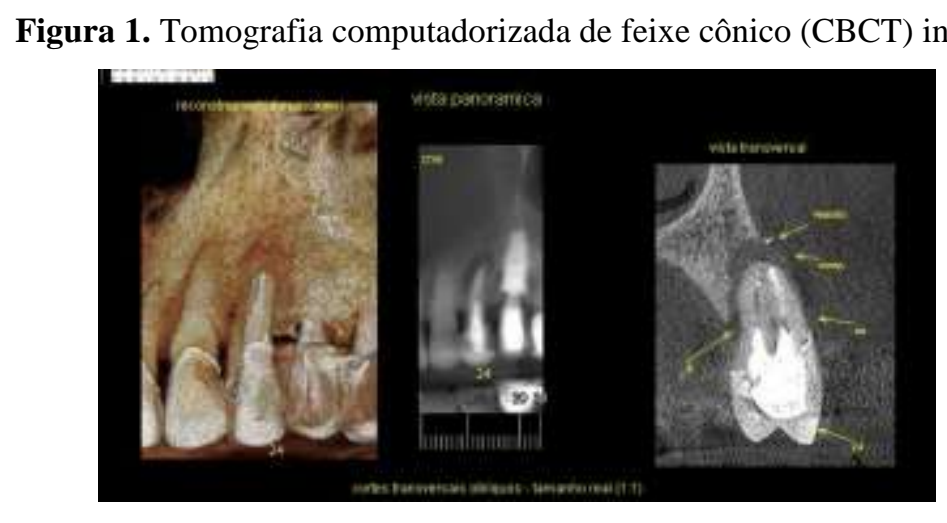

Fonte: Autores.

Previamente ao procedimento cirúrgico, aproximadamente 60 (sessenta minutos) antes, foi adotado um protocolo medicamentoso de 2 (duas) cápsulas de amoxicilina 500mg, 1 (um) comprimido de diazepan 10mg e 1 (um) comprimido de dexametasona $4 \mathrm{mg}$. Foi realizada também a venopunção para coleta do material sanguíneo para obtenção dos agregados plaquetários. $\mathrm{O}$ acesso venoso foi na veia cubital mediana com coleta a vácuo (Figura $2 \mathrm{~A}$ ).Foram coletados dois tubos a vácuo com tampa branca de $9 \mathrm{ml}$ (Vacutube Seco, Biocon ${ }^{\circledR}$, Brasil) e dois tubos a vácuo de tampa vermelha de $9 \mathrm{ml}$ (Labor Import, Brasil). Os 4 tubos foram posicionados em centrífuga digital de rotor fixo e centrifugados por 3 minutos a 2700rpm (Figura 2 B). 
Após esse período a centrífuga foi pausada e os tubos de tampa branca forma retirados da centrífuga. Na sequência a centrifuga foi ligada novamente com os tubos vermelhos sendo centrifugados por mais 10 minutos a $2700 \mathrm{rpm}$. Os tubos brancos foram abertos e com auxílio de uma seringa descartável estéril com agulha o fibrinogênio líquido foi retirado. Os tubos vermelhos foram retirados da centrífuga após 13 minutos e a fibrina foi removida do tubo e separada do restante do material sanguíneo com auxílio de uma tesoura e espátula metálica. As fibrinas foram posicionadas sobre um box metálico para conformação das membranas de L-PRF (Figura 2 C).

Figura 2. (A) Venopunção. (B) Tubos na centrifuga. (C) Fibrina no box.

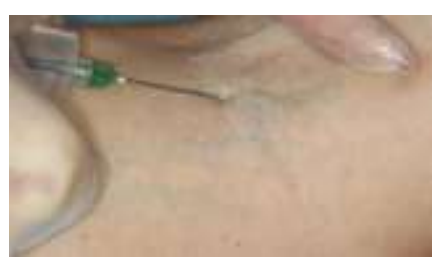

(A)

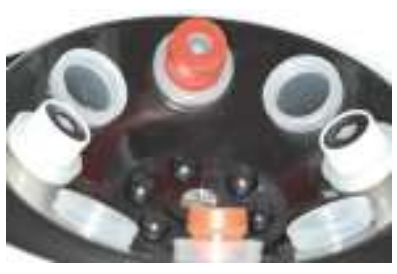

(B)

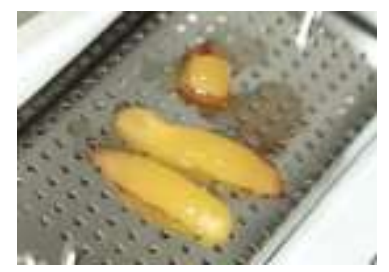

(C)

Fonte: Autores.

Uma membrana foi picotada em fragmentos pequenos e misturada a 0,5g do biomaterial xenógeno Bio-Oss Large (Geistlich

Pharma AG, Wolhusen, Suíça) em uma dappen metálico (Figura 3 A).

Após a mistura entre o L-PRF e o biomaterial xenógeno o fibrinogênio líquido foi gotejado sobre a mistura funcionando como um aglutinante para a mistura (Figura 3B). Vinte minutos de espera e observou-se a formação de um aglomerado contendo biomaterial + L-PRF + fibrinogênio líquido pronto para ser utilizado como substituto ósseo na regeneração óssea (Figura 3 C).

Figura 3. (A) Membrana picotada. (B) Fibrinogênio gotejado. (C) Aglomerado Biomateria+ L-PRF+ fibrinogênio líquido.

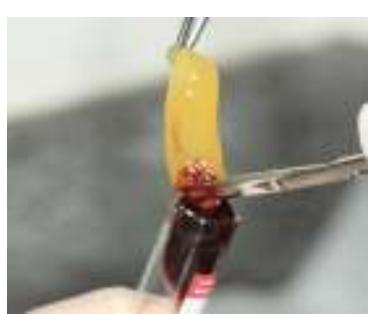

(A)

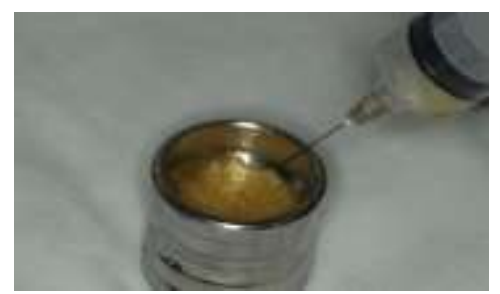

(B)

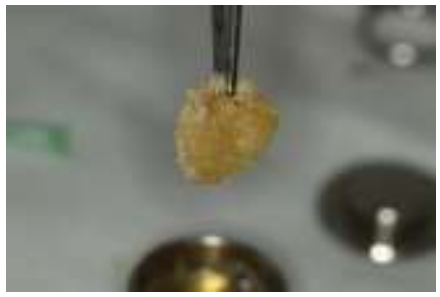

(C)

Fonte: Autores.

Na região do elemento dental condenado 24 (FDI) foi utilizada a técnica anestesia infiltrativa em fundo de vestíbulo com complementação na palatina utilizando anestésico injetável local à base de cloridrato de articaína com epinefrina 1:100.000 (DFL, Rio de Janeiro, RJ, Brasil). Após incisão intrasulcular o elemento dental e o tecido de granulação circundante foram removidos. Sequência de instrumentação recomendadas do fabricante foi realizada para a instalação de implante do tipo cone Morse Indexado Wayfit 3,5x13mm (DSP Biomedical, Campo Largo, PR, Brasil). O implante foi instalado de modo que sua porção cervical ficou $2 \mathrm{~mm}$ abaixo da crista óssea palatina (Figura 4 A). Na porção interna do tecido mole vestibular foi suturada metade de membrana de PRF (L-PRF) (Figura 4 B). Sequencialmente foi posicionado o aglomerado biomaterial+LPRF+Fibrinogênio líquido entre a membrana de L-PRF e o implante instalado (Figura 4 C). A outra metade da membrana de L-PRF foi suturada objetivando um tampão protetor ao implante e a regeneração óssea realizada. 
Figura 4. (A) Implante Instalado. (B) Sutura Membrana L-PRF. (C) Membrana posicionada sobre o implante.

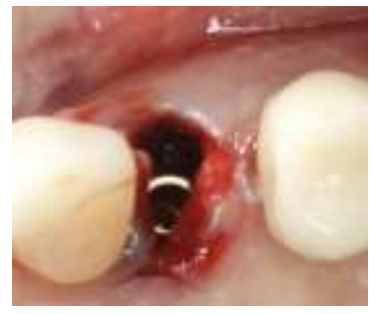

(A)

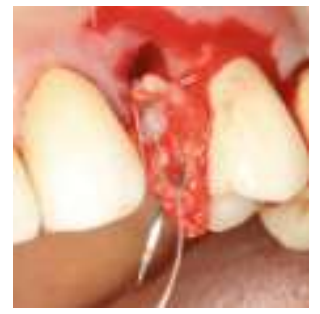

(B)

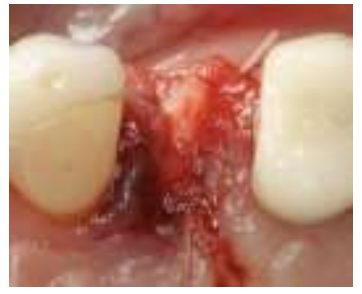

(C)

(A) Fonte: Autores.

Após 6 meses de cicatrização uma incisão foi realizada de modo deslocar conjuntivo da porção palatina para a face vestibular aumentando o volume de conjuntivo na face vestibular . Imediatamente foi realizada a seleção do intermediário protético e instalação do intermediário mini flexcone altura 2,5mm (DSP Biomedical, Campo Largo, PR, Brasil) (Figuras 5 A). Após instalação e torque foi realizada a moldagem para confecção de coroa acrílica provisória. Durante 30 dias foi realizado o condicionamento gengival através da inclusão de Resina flow (Natural Flow A3, DFL, Rio de Janeiro, Brasil) para garantir o correto condicionamento gengival. Após finalização do condicionamento gengival foi realizada nova moldagem e sequente instalação de coroa metalocerâmica sobre o implante (Figura 5 B).

Figura 5. (A) Intermediário Protético. (B) Aspecto final da reabilitação.

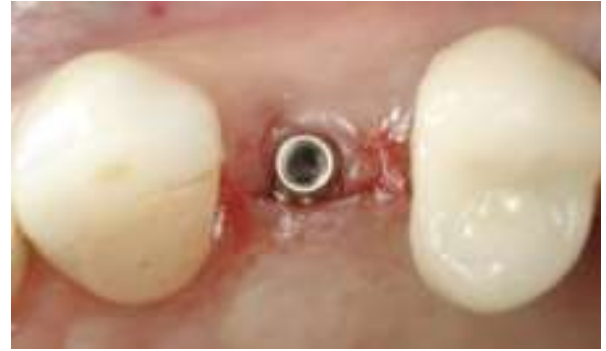

(A)

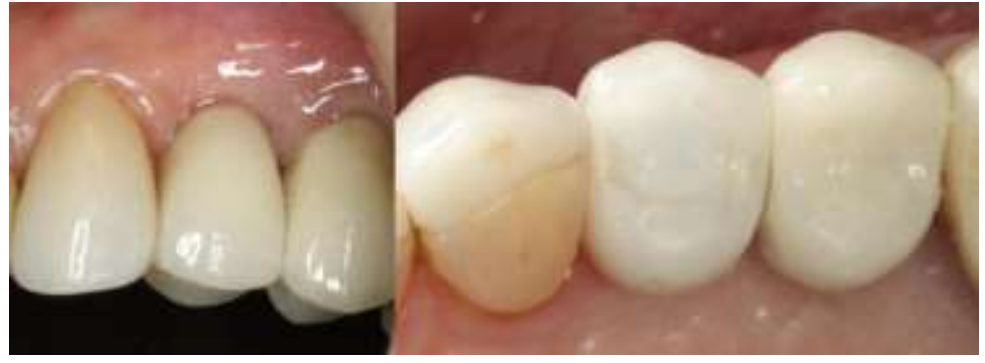

(B)

Fonte: Autores.

Após 6 meses do procedimento cirúrgico foi realizado novas radiografias periapicais e tomografia computadorizada de feixe cônico (CBCT) para acompanhamento da regeneração óssea guiada. (Figura 6).

Figura 6. Tomografia computadorizada após 6 meses.

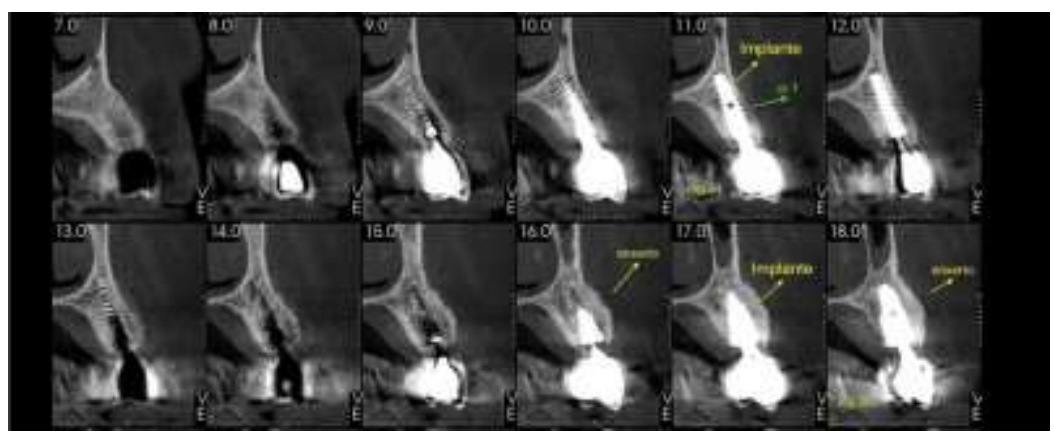

Fonte: Autores. 


\section{Discussão}

Os blocos ósseos autólogos ainda são considerados como o padrão ouro para reconstrução das cristas alveolares reabsorvidas. No entanto, a necessidade de um segundo local cirúrgico provoca uma maior morbidade do paciente além de uma variável reabsorção do enxerto na cicatrização.

O L-PRF em bloco representa uma matriz rica em plaquetas ativadas que secretam uma ampla gama de moléculas bioativas e fatores de crescimento: proteína óssea morfogenética (BMP), fator de crescimento derivado de plaquetas (PDGF), insulina - fator de crescimento semelhante (IGF), fator de crescimento endotelial vascular (VEGF), fator de crescimento transformador- $\beta 1$ (TGF- $\beta 1$ ) e fator de crescimento transformador- $\beta 2$ (TGF- $\beta 2$ ). Estes desempenham papéis-chave na regeneração e regeneração óssea.

Tatulo et al em 2012 mostraram por meio de análise histológica que o PRF reduz o tempo de cicatrização, favorecendo a cicatrização óssea mais rápida.

Revisão sistemática de Canellas et al. 2018, avaliou a utilização de PRF autólogos combinados ou não com outros biomateriais em estudos clínicos controlados randomizados (RCTs). De um total de 1073 artigos identificados um total de 30 ensaios clínicos randomizados preencheram os critérios de inclusão e foram selecionados para análise qualitativa. O PRF mostrou-se mais efetivo na redução de dor e edema pós-operatório em cirurgias de exodontia de terceiro molar. A utilização de PRF associado a biomaterial xenógeno apresentou maior porcentagem osso neoformado. O PRF pode ser utilizado para tratar perfurações da membrana de Schneiderian durante elevação de seio maxilar. Nesta revisão sistemática, a maioria dos estudos confirmou que a PRF diminuiu a reabsorção alveolar após extrações dentárias quando usada sozinha ou combinada com outros biomateriais. Em relação a pacientes fissurados a PRF aumentou significativamente a porcentagem de osso neoformado, mas nenhuma diferença estatística foi observada quando a densidade óssea foi analisada. Os autores acreditavam que a matriz de fibrina pode auxiliar no aumento da formação óssea após a cirurgia. A avaliação do nível ósseo marginal é um dos indicadores mais importantes da saúde do implante. O PRF é um biomaterial promissor para melhorar a reconstrução da fenda alveolar e reduzir as taxas de reabsorção óssea marginal peri-implantar. O efeito do PRF no aumento do seio não mostrou nenhuma vantagem na porcentagem de formação óssea final, entretanto o tempo de cicatrização pode ser reduzido quando o PRF foi adicionado

As principais vantagens do uso de PRF são o baixo custo, a facilidade de obtenção e a total biocompatibilidade. Além disso a fibrina rica em plaquetas e leucócitos atua como fonte de fatores de crescimento aumentando a vascularização e a diferenciação osteoblástica. Os diversos protocolos de preparo e as lacunas no conhecimento de suas propriedades biológicas ainda dificultam a interpretação dos resultados clínicos.

\section{Considerações Finais}

O uso do fibrina rica em plaquetas e leucócitos (L-PRF) em procedimentos de regeneração óssea guiada associado a biomaterial xenógeno apresenta resultado satisfatório no ganho de volume ósseo favorecendo a manutenção dos tecidos adjacentes a reabilitação implanto suportada.

Mais estudos clínicos com desenhos metodológicos adequados são necessários para confirmação de seus benefícios.

\section{Referências}

Benic, G. I. \& Hämmerle, C. H. (2014). Horizontal bone augmentation by means of guided bone regeneration. Journal Periodontol 2000. 66(1):13-40. doi: $10.1111 /$ prd.12039.

Boswell, S. G., Cole, B. J., Sundman, E. A., Karas, V. \& Fortier, L. A. (2012). Platelet-rich plasma: a milieu of bioactive factors. Arthroscopy. $28: 429-39$. 
Canellas, J. V. D. S., Medeiros, P. J. D., Figueredo, C. M. D. S., Fischer, R. G. \& Ritto, F. G. (2018). Platelet-rich fibrin in oral surgical procedures: a systematic review and meta-analysis. Int J Oral Maxillofac Surg. pii: S0901-5027(18)30255-8. doi: 10.1016/j.ijom.2018.07.007.

Choukroun, J., Adda, F., Schoeffler, C. \& Vervelle, A. (2000). Une opportunite' en paro-implantologie: Le PRF. Implantodontie. 42:55-62.

Cortellini, S., Castro, A. B., Temmerman, A., Van, Dessel, J., Pinto, N., Jacobs, R. \& Quirynen, M. (2018). Leucocyte and plateletrich fibrin block for bone augmentation procedure: A proof-of-concept study. J Clin Periodontol. 45(5):624-34. doi: 10.1111/jcpe.12877.

Dohan, D. M., Choukroun, J., Diss, A., Dohan, S. L., Dohan, A. J., Mouhyi, J., Gogly, B. (2006). Platelet-rich fibrin (PRF): a second-generation platelet concentrate. Part II: platelet-related biologic features. Oral Surg Oral Med Oral Pathol Oral Radiol Endod. 101(3):e45-50.

Dohan, D. M., Choukroun, J., Diss, A., Dohan, S. L., Dohan, A. J., Mouhyi, J., Gogly, B. (2006). Platelet-rich fibrin (PRF): a second-generation platelet concentrate. Part III: leucocyte activation: a new feature for platelet concentrates? Oral Surg Oral Med Oral Pathol Oral Radiol Endod. 101(3):e51-5.

Dohan, Ehrenfest, D. M., Bielecki, T., Jimbo, R., Barbe, G., Del Corso, M., Inchingolo, F. \& Sammartino, G. (2012). Do the fibrin architecture and leukocyte content influence the growth factor release of platelet concentrates? An evidence-based answer comparing a pure platelet-rich plasma (P-PRP) gel and a leukocyte- and platelet-rich fibrin (L-PRF). Curr Pharm Biotechnol. 13:1145-52.

Dohan Ehrenfest, D. M., Del Corso, M., Diss, A., Mouhyi, J. \& Charrier, J. B. (2010). Three-dimensional architecture and cell composition of a Choukroun's platelet-rich fibrin clot and membrane. J Periodontol. 81(4):546- 55.

Dohan Ehrenfest, D. M., Rasmusson, L., Albrektsson, T. (2009). Classification of platelet concentrates: from pure platelet-rich plasma (P- PRP) to leucocyteand platelet-rich fibrin (L-PRF). Trends Biotechnol. 27 (3):158-67.

Donos, N., Mardas, N. \& Chadha, V. (2008). Clinical outcomes of implants following lateral bone augmentation: systematicassessment of available options (barrier membranes, bone grafts, split osteotomy). J Clin Periodontol. (2008) Sep;35(8 Suppl):173-202. doi: 10.1111/j.1600-051X.2008.01269.x.

Fujioka-Kobayashi, M., Miron, R. J., Hernandez, M., Kandalam, U., Zhang, Y. \& Choukroun, J. (2017). Optimized platelet-rich fibrin with the low-speed concept: growth factor release, biocompatibility, and cellular response. J Periodontol. 88(1):112-21.

Kobayashi, E., Fluckiger, L., Fujioka-Kobayashi, M., Sawada, K., Sculean, A., Schaller, B. \& Miron, R. J. (2016). Comparative release of growth factors from PRP, PRF, and advanced-PRF. Clin Oral Investig. 20(9):2353-60.

Mourão, C. F., Valiense, H., Melo, E. R., Mourão, N. B. \& Maia, M. D. (2015). Obtention of injectable platelets rich-fibrin (i-PRF) and its polymerization with bonegraft: technical note. Rev Col Bras Cir. Nov-Dec. 42(6):421-3. doi: 10.1590/0100-69912015006013.

Nkenke, E. \& Neukam, F. W. (2014). Autogenous bone harvesting and grafting in advanced jaw resorption: morbidity, resorption and implant survival. Eur J Oral Implantol. Summer;7 Suppl 2:S203-17.

Oncu, E. \& Kaymaz, E. (2017). Assessment of the effectiveness of platelet rich fibrin in the treatment of Schneiderian membrane perforation. Clin Implant Dent Relat Res. 19 (6):1009-14.

Pirraco, R. P., Reis. R. L., Marques, A. P. (2013). Effect of monocytes/macrophages on the early osteogenic differentiation of hBMSCs. J Tissue Eng Regen Med. 7(5):392-400.

Schär, M. O., Diaz-Romero, J., Kohl, S., Zumstein, M. A., Nesic, D. (2015). Platelet-rich concentrates differentially release growth factors and induce cellmigration in vitro. Clin Orthop Relat Res. 473(5):1635-43. doi: 10.1007/s11999-015-4192-2.

Simonpieri, A., Del Corso, M., Sammartino, G., Dohan Ehrenfest, D. M. (2009). The relevance of Choukroun's platelet-rich fibrin and metronidazole during complexmaxillary rehabilitations using bone allograft. Part I: a new grafting protocol. Implant Dent. 18(2):102-11. doi: 10.1097/ID.0b013e318198cf00.

Soldatos, N. K., Stylianou, P., Koidou, V. P., Angelov, N., Yukna, R., Romanos, G. E. (2017). Limitations and options using resorbable versus nonresorbable membranes for successful guided bone regeneration. Quintessence Int. 48(2):131-47. doi: 10.3290/j.qi.a37133.

Tatullo, M., Marrelli, M., Cassetta, M., Pacifici, A., Stefanelli, L. V., Scacco, S., Dipalma, G., Pacifici, L. \& Inchingolo, F. (2012). Platelet Rich Fibrin (P.R.F.) in reconstructive surgery of atrophied maxillary bones: clinical and histological evaluations. Int J Med Sci. 9 (10):872-80.

Zhang, Y., Tangl, S., Huber, C. D., Lin, Y., Qiu, L. \& Rausch-Fan, X. (2012). Effects of Choukroun's plate- let-rich fibrin on bone regeneration in combination with deproteinized bovine bone mineral in maxillary sinus augmentation: a histological and histomorphometric study. J Craniomaxillofac Surg. 40(4):321-8. 